\title{
Pre-pectoral one-stage breast reconstruction with anterior biological acellular dermal matrix coverage
}

\author{
Ayesha Khan, Marios-Konstantinos Tasoulis^, Victoria Teoh, Aleksandra Tanska, Ruth Edmonds, \\ Gerald Gui
}

Breast Surgery Unit, The Royal Marsden NHS Foundation Trust, London, UK

Contributions: (I) Conception and design: All authors; (II) Administrative support: All authors; (III) Provision of study materials or patients: All authors; (IV) Collection and assembly of data: All authors; (V) Data analysis and interpretation: A Khan, MK Tasoulis, G Gui; (VI) Manuscript writing: All authors; (VII) Final approval of manuscript: All authors.

Correspondence to: Dr. Marios-Konstantinos Tasoulis, MD, PhD, FEBS (Breast), FRCS. Breast Surgery Unit, The Royal Marsden NHS Foundation Trust, Fulham Road, SW3 6JJ, London, UK. Email: mtasoulis@med.uoa.gr.

Background Pre-pectoral implant breast reconstruction (IBR) is gaining popularity. Several techniques using different types of meshes and methods of placement have been described, but no method is currently considered standard. The aim of this study was to evaluate the outcomes of pre-pectoral IBR using acellular dermal matrix (ADM) for anterior implant cover.

Methods: Retrospective cohort study of consecutive patients who underwent pre-pectoral IBR between November 2016 to August 2018. Data on demographics, adjuvant therapies and operative technique was collected. Postoperative complications, length of hospital stay and secondary cosmetic procedures were recorded. Statistical analysis was performed using descriptive statistics, non-parametric tests and logistic regression.

Results: One hundred and eleven pre-pectoral IBR were performed in 65 patients. Median age was 41 [interquartile range (IQR), 35-51.5] years, and BMI 22 (IQR, 20.4-24.4) kg/m². Therapeutic mastectomy was performed in 33 procedures with nipples preservation in 78 cases. The median mastectomy weight and implant volume was 360 (IQR, 220-533) gr, and 445 (IQR, 400-475) cc respectively. At a median followup of 18 (IQR, 12-22.5) months, 37 mastectomies had at least 1 complication, but only 12 required surgery. The implant loss rate was $4.5 \%$ ( 5 cases). Lipofilling as secondary procedure was performed in $10.8 \%$ of cases. Factors associated with post-operative complications on univariate analysis were nipple preservation $(\mathrm{P}=0.028), \mathrm{BMI}(\mathrm{P}=0.01)$ and implant volume $(\mathrm{P}=0.027)$ but these did not remain significant on multivariate analysis.

Conclusions: Pre-pectoral IBR using ADM for anterior implant cover is associated with low complication and reconstructive failure rate. Patient selection and meticulous surgical technique are important for successful outcome.

Keywords: Breast reconstruction; implant; pre-pectoral; acellular dermal matrix (ADM); implant loss

Submitted Aug 01, 2020. Accepted for publication Jan 12, 2021.

doi: $10.21037 /$ gs-20-652

View this article at: http://dx.doi.org/10.21037/gs-20-652

^ ORCID: 0000-0001-9453-1547. 


\section{Introduction}

Breast reconstruction with the use of implants is the most commonly employed method to restore the breast mound after mastectomy, accounting for approximately $80 \%$ of the cases in both the United Kingdom and United States of America (1,2). Traditionally, implant-based breast reconstruction (IBR) involved lifting of the pectoralis major muscle and insertion of the implant in the sub-pectoral pocket, as well as use of the serratus fascia with or without the rectus abdominis to provide complete implant cover. The introduction of acellular dermal matrices (ADMs) and their use as a hammock to augment the inferior-lateral sub-pectoral pocket revolutionised the practice and allowed one-stage, direct to implant (DTI) breast reconstruction.

With the proven safety and widespread use of ADMs (3-5), techniques have evolved and there has been a shift in the last few years towards pre-pectoral breast reconstruction (6). A number of methods have been described using different types of meshes (synthetic or biological ADMs) to facilitate DTI pre-pectoral IBR. These methods include pre-shaped ADMs fully covering the implant and being secured on the chest wall $(3,7)$ as well as a number of variations of mesh use to wrap the whole implant $(8,9)$ or cover part of it $(10-12)$. So far, there has not been any conclusive data suggesting superiority of a single, standard technique and the reported outcomes may vary (13).

The aim of this study was to present the outcomes of DTI pre-pectoral IBR using an ADM to cover only the anterior surface of the implant in a consecutive cohort of patients treated in a high-volume tertiary referral breast unit. We present the following article in accordance with the STROBE reporting checklist (available at http://dx.doi. org/10.21037/gs-20-652).

\section{Methods}

This was a retrospective cohort study of patients who underwent DTI pre-pectoral IBR under the care of the senior author (GG) between November 2016 and August 2018. Cases were identified from a prospectively collected database. Collected data included patient demographics (age, BMI, smoking history), adjuvant therapies and operative technique (nipple sparing/skin sparing mastectomy, implant size, incision). All postoperative complications, length of stay and secondary cosmetic procedures were recorded during the follow-up period which was in accordance with our unit's policy. Surgical complications included infection, inflammatory skin reaction (erythema), haematoma, seroma, skin necrosis, nipple necrosis, and implant loss. Secondary revision surgery mainly included fat grafting to improve the aesthetic outcome following shared decision making with the patients and also nipple reconstruction in women undergoing nipple-sacrificing mastectomy. Data collection bias was limited by the inclusion of all consecutive patients who underwent DTI pre-pectoral IBR during the predetermined timeframe.

All procedures performed in the study were in accordance with the ethical standards of the institutional and national research committee and with the 1964 Helsinki declaration (as revised in 2013). The study was reviewed and approved by the Trust's Clinical Audit Committee (BR160) and the data were collected and kept in accordance with The Data Protection Act (UK), the International Conference on Harmonization (ICH) Guidelines for Good Clinical Practice (GCP) and the Trust's Standard Operating Procedures. The guidance from the Strengthening the Reporting of Observational Studies in Epidemiology (STROBE) statement was applied (14).

\section{Surgical technique-implant and ADM reconstruction}

All mastectomies and reconstructions were performed using a uniform technique by, or under the direct supervision of, the senior author (GG). A skin or nipple sparing mastectomy was performed using the hydrodissection technique and scissors as previously described (15). A bovine ADM was used to hold the implant in position and create the pre-pectoral pocket. Before the introduction of the meshed ADM (SurgiMend ${ }^{\circledR}$, PRS Meshed, Integra Life Sciences, New Jersey, USA) a sheet ADM was used (SurgiMend ${ }^{\circledR}$, PRS $^{\mathrm{TM}}$, Integra Life Sciences, New Jersey, USA) with the creation of large fenestrated pores intra-operatively to achieve a meshed device. The ADM was prepared for use prior to surgery following manufacturer instructions. A single piece of meshed ADM, trimmed and tailored to create the pre-pectoral pocket, covering only the anterior surface of the implant, was secured using absorbable polydioxanone $\left(\mathrm{PDS}^{\circledR} \mathrm{II}\right.$, Ethicon US, LLC) sutures. Up to three interrupted sutures were placed around the periphery of each of the superior, lateral, medial and inferior edges, suturing the $\mathrm{ADM}$ to the pectoralis fascia along the original mastectomy footprint. The sutures were placed through the spaces in the mesh or through the ADM substance itself, with the knots facing downwards and thus being covered by the ADM. The lateral position of the ADM was used to control the cleavage position of the implant. The inferior edge of 

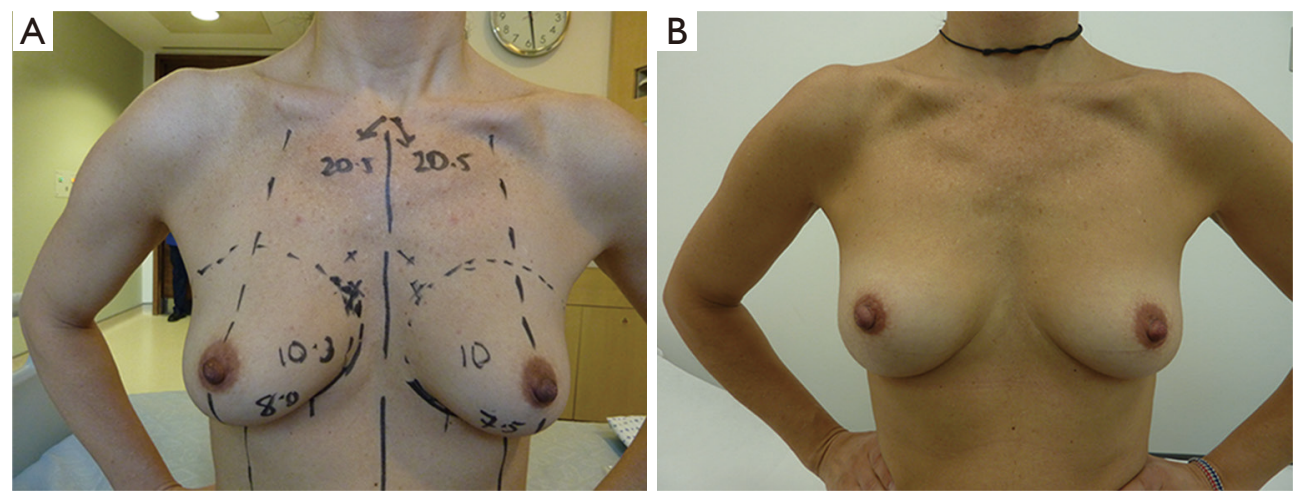

Figure 1 Cosmetic outcome after unilateral nipple and skin sparing mastectomy and pre-pectoral IBR in a woman with right breast cancer. (A) Pre-operative; (B) post-operative. IBR, implant breast reconstruction.

the $\mathrm{ADM}$ was the aperture through which the implant was placed for central and inframammary crease incisions. For lateral incisions, the superior, medial and inferior surfaces were closed first, leaving the lateral aperture as the access site. Implants were inserted using accepted measures to minimize the risk of infection (including thorough pocket lavage with sterile water and betadine, the minimal handling of implants, change of surgical gloves prior to handling prostheses and theatre laminar air flow). All reconstructions were performed with textured silicone implants.

\section{Primary objective}

The primary objective of the study was to evaluate the surgical outcomes after DTI pre-pectoral IBR including:

(I) Surgical complications;

(II) Incidence of surgical interventions for complications and secondary cosmetic procedures;

(III) Overall length of hospital stay.

\section{Secondary objective}

The secondary objective of the study was to try to identify factors that may be associated with the development of complications. These factors included age, BMI, smoking status, nipple preservation, incision type, implant volume, ADM type (fenestrated intra-operatively or pre-meshed) and previous or adjuvant radiotherapy.

\section{Statistical analysis}

Complication rates and surgical outcomes are presented using simple descriptive statistics. For categorical variables absolute numbers and corresponding percentages and for continuous variables median and interquartile range (IQR) was used. Non-parametric tests (chi-square and MannWhitney $\mathrm{U}$ test as appropriate) were performed to assess potential associations between patient demographic and surgical procedural variables and the development of postoperative complications, and logistic regression was used for multivariate analysis. Patients with missing data on primary outcome measures were excluded from the analysis. All statistical analyses were performed with the use of SPSS v.23 (IBM SPSS Statistics for Windows; IBM Corp., Armonk, NY, USA).

\section{Results}

During the study period 111 pre-pectoral breast reconstructions were performed in 65 patients. All patients had complete datasets for primary outcomes variables and none were excluded from the analysis. The median patient age was 41 (IQR, 35-51.5) years and the median BMI was 22 (IQR, 20.4-24.4) $\mathrm{kg} / \mathrm{m}^{2}$. Only 4 patients were smokers (6.2\%). Eleven patients (16.9\%) had a prior history of breast cancer and 3 (4.6\%) had previously received radiotherapy.

Thirty-one patients had a breast cancer diagnosis and underwent 33 therapeutic mastectomies (2 patients had bilateral cancer) (Figure 1) whilst the remainder 78 mastectomies were performed as risk reducing procedures in patients with either contralateral breast cancer or with high genetic predisposition (Figure 2). The tumour characteristics are presented in Table 1. Axillary surgery in the form of sentinel lymph node biopsy (SLNB) was performed in all therapeutic mastectomies $(n=33)$ and was positive in only 2 cases which had further axillary 

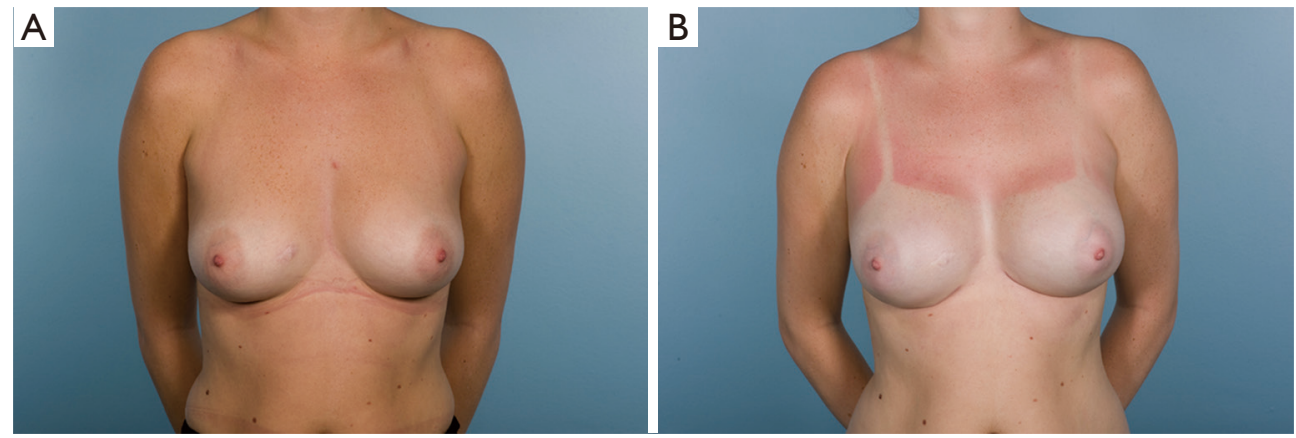

Figure 2 Cosmetic outcome after bilateral nipple and skin sparing mastectomy and pre-pectoral IBR in a woman with $B R C A 2$ gene mutation. (A) Pre-operative; (B) post-operative. IBR, implant breast reconstruction.

lymph node dissection (ALND). Adjuvant chemotherapy was administered in 4 patients $(6.2 \%), 2$ of whom also received endocrine therapy. Adjuvant hormone therapy only was prescribed in 14 patients and $2(3.1 \%)$ received postmastectomy radiotherapy.

Procedural details are presented in Table 2. The nipple was preserved in 78 mastectomies. The median mastectomy weight was 360 (IQR, 220-533) gr and the median volume of the implant used was 445 (IQR, 400-475) cc. The median follow-up was 18 (IQR, 12-22.5) months. Thirtyseven mastectomies were associated with at least one complication. Details are presented in Table 3. A total of $12(10.8 \%)$ mastectomies required additional intervention because of the complications. In 3 cases this involved evacuation of haematoma. Surgical debridement for wound dehiscence was required in 1 case and there was also one return to theatre for persistent inflammatory skin reaction but no infection was identified.

Mastectomy flap necrosis is a major factor impacting on poor outcome after IBR. Whilst empirically this may seem to be an important consideration in the pre-pectoral approach, the implant loss rates are still within the acceptable boundaries achieved in the sub-pectoral DTI era. In this study, 7 cases developed skin flap necrosis leading to removal of the implant in 3 cases, and one conversion to a tissue expander. Nipple preservation is also a risk factor for ischaemia of the overlying skin envelope; although there was only one patient with full thickness nipple necrosis, this resulted in an implant loss that might have been avoided in a submuscular placement where the pectoral muscle often provides an intervening protective separating the implant pocket.

The risk of surgical complications was higher in patients who underwent nipple-sparing compared to nipple- sacrificing mastectomy $(\mathrm{P}=0.028)$. Factors associated with post-operative complications on univariate analysis were patient $\mathrm{BMI}(\mathrm{P}=0.01)$ and larger implant volume $(\mathrm{P}=0.027)$. Although certain incision types like $\mathrm{J}$ incision with nipple preservation were associated with a numerically higher complication rate, this did not reach statistical significance $(\mathrm{P}=0.083)$. None of these variables were found to be associated with the risk of post-operative complications on multivariate regression analysis. The median length of hospital stay was 2 (IQR, 1-3) days. Secondary procedures in the form of lipofilling were performed in 12 cases (10.8\%); one session in 7 and 2 sessions in 5 cases (Figure 3).

\section{Discussion}

Improved surgical appreciation of the mastectomy oncoplastic plane and technological advances in the quality of ADMs, have led to increased popularity of pre-pectoral DTI IBR among breast reconstructive surgeons as an alternative technique to sub-pectoral implant placement $(6,16,17)$. Several methods of pre-pectoral DTI IBR have been described. These include the use of pre-shaped ADMs $(3,7)$ as well as use of $\mathrm{ADMs}$ to fully wrap $(8,9)$ or cover part of a fixed volume or expander implant (10-12). This heterogeneity in techniques, could at least in part, explain the variable outcomes reported in a recent systematic review (13).

In the present study, $\mathrm{ADM}$ (sheet or meshed) was used to provide anterior implant coverage and secure the implant position in the pre-pectoral pocket. The overall postoperative complication rate was $33.3 \%$. This is comparable to the published literature (13). Although this included all major and minor complications, for example non-clinically significant seromas requiring no intervention, it suggests there is room for improvement. Surgical intervention 
Table 1 Tumour characteristics

\begin{tabular}{|c|c|}
\hline Tumour characteristics & Number of mastectomies, $n=33$ \\
\hline Invasive cancer & 24 \\
\hline IDC & 17 \\
\hline ILC & 4 \\
\hline Mixed IDC and ILC & 2 \\
\hline Other $^{\dagger}$ & 1 \\
\hline \multicolumn{2}{|l|}{ Grade of invasive cancer } \\
\hline Grade I & 5 \\
\hline Grade ॥ & 13 \\
\hline Grade III & 6 \\
\hline $\mathrm{DCIS}^{\ddagger}$ & 23 \\
\hline \multicolumn{2}{|l|}{ Grade of DCIS } \\
\hline Low grade & 4 \\
\hline Intermediate grade & 4 \\
\hline High grade & 15 \\
\hline \multicolumn{2}{|l|}{ ER status } \\
\hline Negative & 5 \\
\hline Positive & 18 \\
\hline \multicolumn{2}{|l|}{ PgR status } \\
\hline Negative & 6 \\
\hline Positive & 17 \\
\hline \multicolumn{2}{|l|}{ HER2 status } \\
\hline Negative & 23 \\
\hline Equivocal & 0 \\
\hline Positive & 0 \\
\hline
\end{tabular}

Data presented per breast. ${ }^{\dagger}$, follicular lymphoma, no grade or receptor status associated with the lymphoma; ${ }^{\ddagger}$, nine cases had pure DCIS without invasive disease. IDC, invasive ductal carcinoma; ILC, invasive lobular carcinoma; DCIS, ductal carcinoma in situ; ER, oestrogen receptor; PgR, progesterone receptor; HER2, human epidermal growth factor receptor 2.

because of a complication was required in only $10.8 \%$ of the cases. This is similar to a recent large multicentre study using pre-shaped $\mathrm{ADM}$, which reported that $16.7 \%$ of the cases required surgical exploration (3) while another study assessing the use of $\mathrm{ADM}$ for anterior implant cover showed a $22.2 \%$ complication rate requiring further surgery (11). In the present cohort, women underwent mastectomy using the hydrodissection technique. Although this technique may be
Table 2 Surgical procedure details

\begin{tabular}{lc}
\hline Procedural details & $\begin{array}{c}\text { Number of mastectomies, } \\
\mathrm{n}=111\end{array}$ \\
\hline Incision type & 22 \\
Transverse with excision of NAC & 68 \\
IMC preserving NAC & 11 \\
Wise pattern with excision of NAC & 6 \\
Wise pattern preserving the NAC & 4 \\
J preserving the NAC & 67 \\
ADM type & 44 \\
Meshed & 360 [220-533] \\
Sheet & 445 [400-475] \\
Mastectomy weight (gr), median [IQR] & \\
Implant volume (cC), median [IQR] & \\
\hline NAC, nipple areola complex; IMC, inframammary crease; IQR, \\
interquartile range.
\end{tabular}

Table 3 List of surgical complications

\begin{tabular}{lc}
\hline Surgical complications & $\begin{array}{c}\text { Number of mastectomies (\%), } \\
\mathrm{n}=111\end{array}$ \\
\hline At least on complication & $37(33.3)$ \\
Infection & $9(8.1)$ \\
Inflammatory skin reaction & $6(5.4)$ \\
(erythema) & $3(2.7)$ \\
Haematoma & $7(6.3)$ \\
Seroma & $5(4.5)$ \\
Implant loss & $7(6.3)$ \\
Skin flap necrosis & $7(9.0)^{\dagger}$ \\
Superficial nipple necrosis & $1(1.3)^{\dagger}$ \\
Full thickness nipple necrosis & $12(10.8)$ \\
Dehiscence & $4(3.6)$ \\
Capsule formation
\end{tabular}

${ }^{\dagger}$, the percentage of nipple necrosis was calculated based on the number of nipple-sparing mastectomies $n=78$.

associated with increased risk of skin flap complications, the observed skin flap necrosis rate was $6.3 \%$, which is similar to the published literature $(3,13,16)$. The assessment of skin flaps in the present study was performed by means of visual inspection and evaluation of perfusion based on clinical 

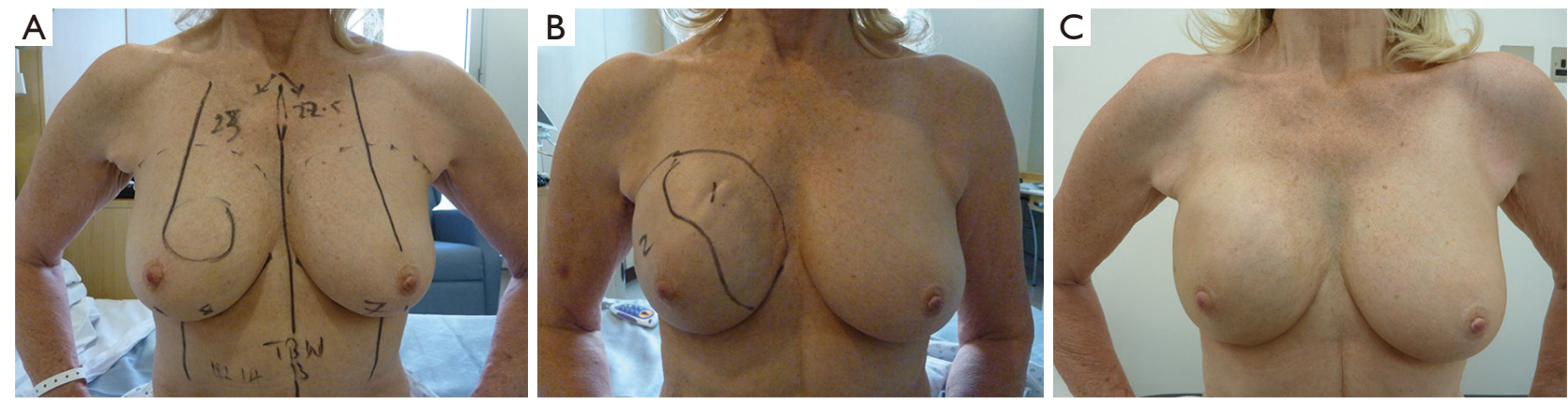

Figure 3 Cosmetic outcome after unilateral nipple and skin sparing mastectomy and pre-pectoral breast reconstruction with subsequent fat grafting in a woman with a right breast cancer. (A) Pre-operative; (B) post-operative after pre-pectoral IBR; (C) post-operative after fat grafting. IBR, implant breast reconstruction.

judgement (warmth, colour, capillary refill). Although we did not use peri-operative assessment of vascular flow to the mastectomy flaps, the use of such techniques in case of concern may provide useful information to guide the surgical plan (18). The implant loss rate in the present study was $4.5 \%$. This compares favourably to the pooled implant loss rate of pre-pectoral IBR with use of ADM (7.2\%) (13) and is similar to that reported in other studies using ADM only for anterior implant coverage $(5.5-6.7 \%)(10,11,19)$.

In pre-pectoral IBR, the pectoralis major is not lifted and therefore is not available to provide the extra layer of soft tissue to cover the implant. This may result in more visible implant contour and rippling requiring fat transfer to address these irregularities. In the present study, 10.8\% of the cases underwent secondary fat grafting. This is low compared to other studies using a similar $\mathrm{ADM}$ technique, reporting autologous fat transfer rates ranging between $16.7-38 \%(10,11,19)$. A potential explanation might be the use of hydrodissection in the present cohort. This technique is considered to provide more even mastectomy flaps, potentially associated with lower autologous fat transfer rates. These early results should be interpreted with caution as our previous experience with hydrodissection was assessed in sub-pectoral IBR (15). Alternatives techniques to improve the upper pole aesthetics and potentially decrease visibility of the implant contour and rippling have also been described, using anterior implant cover with $\mathrm{ADM}$ and a "slip" of the pectoralis muscle for additional superior coverage (12).

Appropriate patient selection and meticulous surgical technique should be employed to reduce risks and optimize outcomes. High BMI $(3,20,21)$ and increased implant volume (3) have been shown to be associated with the risk of complications, while the data on radiotherapy are conflicting
$(20,22)$. In the present study BMI and implant volume were associated with higher post-operative complication rate but these did not remain significant in multivariate analysis, as previously described in other studies $(3,21)$. Radiotherapy did not have a detrimental effect in our study, but cautious interpretation of the data is required because of the small number of patients receiving radiotherapy with corresponding very low event rates, as a result of patient selection.

The present study has a number of limitations. This is a retrospective analysis of a prospectively collected database describing the practice of a single surgeon in a large tertiary referral centre. In addition, although the median study follow-up is 18 months, this might not be enough to capture all secondary revision surgery that might be required in the context of DTI pre-pectoral IBR without data on long-term outcomes.

\section{Conclusions}

Single-stage, DTI pre-pectoral IBR with use of a biological ADM for anterior implant cover appears to be a safe technique. The results of the present study demonstrate that the post-operative complication profile and need for revision surgery are comparable to other pre-pectoral IBR techniques. Careful patient selection, meticulous surgical technique and experience are of paramount importance to achieve optimal outcomes.

\section{Acknowledgments}

Part of the data presented as poster at the 20th Annual Meeting of the American Society of Breast Surgeons in Dallas, TX, USA, April 30 to May 05, 2019. The authors 
would like to acknowledge the National Institute for Health Research Biomedical Research Centre (NIHR-BRC) for supporting this study.

Funding: None.

\section{Footnote}

Reporting Checklist: The authors have completed the STROBE reporting checklist. Available at http://dx.doi. org/10.21037/gs-20-652

Data Sharing Statement: Available at http://dx.doi. org/10.21037/gs-20-652

Peer Review File: Available at http://dx.doi.org/10.21037/gs20-652

Conflicts of Interest: All authors have completed the ICMJE uniform disclosure form (available at http://dx.doi. org/10.21037/gs-20-652). GG serves as a consultant for Integra Life Science on an ad hoc basis. The other authors have no conflicts of interest to declare.

Ethical Statement: The authors are accountable for all aspects of the work in ensuring that questions related to the accuracy or integrity of any part of the work are appropriately investigated and resolved. The study was conducted in accordance with the Declaration of Helsinki (as revised in 2013). The study reviewed and approved by the Trust's Clinical Audit Committee (BR160) and the data were collected and kept in accordance with the Data Protection Act (UK), the International Conference on Harmonization (ICH) Guidelines for Good Clinical Practice (GCP) and the Trust's Standard Operating Procedures. Individual consent for this retrospective analysis was waived.

Open Access Statement: This is an Open Access article distributed in accordance with the Creative Commons Attribution-NonCommercial-NoDerivs 4.0 International License (CC BY-NC-ND 4.0), which permits the noncommercial replication and distribution of the article with the strict proviso that no changes or edits are made and the original work is properly cited (including links to both the formal publication through the relevant DOI and the license). See: https://creativecommons.org/licenses/by-nc-nd/4.0/.

\section{References}

1. American Society of Plastic Surgeons. Plastic surgery statistics report. ASPS National Clearinghouse of Plastic Surgery Procedural Statistics 2019. 2019.

2. Leff DR, Bottle A, Mayer E, et al. Trends in Immediate Postmastectomy Breast Reconstruction in the United Kingdom. Plast Reconstr Surg Glob Open 2015;3:e507.

3. Chandarana M, Harries S; National Braxon Audit Study Group. Multicentre study of prepectoral breast reconstruction using acellular dermal matrix. BJS Open 2020;4:71-7.

4. Tasoulis MK, Teoh V, Khan A, et al. Acellular dermal matrices as an adjunct to implant breast reconstruction: analysis of outcomes and complications. Eur J Surg Oncol 2020;46:511-5.

5. Potter S, Chambers A, Govindajulu S, et al. Early complications and implant loss in implant-based breast reconstruction with and without acellular dermal matrix (Tecnoss Protexa®): a comparative study. Eur J Surg Oncol 2015;41:113-9.

6. Tasoulis MK, Iqbal FM, Cawthorn S, et al. Subcutaneous implant breast reconstruction: time to reconsider? Eur J Surg Oncol 2017;43:1636-46.

7. Vidya R, Cawthorn SJ. Muscle-sparing ADM-assisted breast reconstruction technique using complete breast implant coverage: a dual-institute UK-based experience. Breast Care (Basel) 2017;12:251-4.

8. Lee JS, Kim JS, Lee JH, et al. Prepectoral breast reconstruction with complete implant coverage using double-crossed acellular dermal matrixs. Gland Surg 2019;8:748-57.

9. Reitsamer R, Peintinger F. Prepectoral implant placement and complete coverage with porcine acellular dermal matrix: a new technique for direct-to-implant breast reconstruction after nipple-sparing mastectomy. J Plast Reconstr Aesthet Surg 2015;68:162-7.

10. Jones G, Yoo A, King V, et al. Prepectoral immediate direct-to-implant breast reconstruction with anterior AlloDerm coverage. Plast Reconstr Surg 2017;140:31S-8S.

11. Paydar KZ, Wirth GA, Mowlds DS. Prepectoral breast reconstruction with fenestrated acellular dermal matrix: a novel design. Plast Reconstr Surg Glob Open 2018;6:e1712.

12. Pittman TA, Abbate OA, Economides JM. The P1 
method: prepectoral breast reconstruction to minimize the palpable implant edge and upper pole rippling. Ann Plast Surg 2018;80:487-92.

13. Wagner RD, Braun TL, Zhu H, et al. A systematic review of complications in prepectoral breast reconstruction. J Plast Reconstr Aesthet Surg 2019;72:1051-9.

14. von Elm E, Altman DG, Egger M, et al. Strengthening the Reporting of Observational Studies in Epidemiology (STROBE) statement: guidelines for reporting observational studies. BMJ 2007;335:806-8.

15. Tasoulis MK, Agusti A, Karakatsanis A, et al. The use of hydrodissection in nipple- and skin-sparing mastectomy: a retrospective cohort study. Plast Reconstr Surg Glob Open 2019;7:e2495.

16. Nealon KP, Weitzman RE, Sobti N, et al. Prepectoral direct-to-implant breast reconstruction: safety outcome endpoints and delineation of risk factors. Plast Reconstr Surg 2020;145:898e-908e.

17. Li L, Su Y, Xiu B, et al. Comparison of prepectoral and subpectoral breast reconstruction after mastectomies: a

Cite this article as: Khan A, Tasoulis MK, Teoh V, Tanska A, Edmonds R, Gui G. Pre-pectoral one-stage breast reconstruction with anterior biological acellular dermal matrix coverage. Gland Surg 2021;10(3):1002-1009. doi: 10.21037/gs-20-652 systematic review and meta analysis. Eur J Surg Oncol 2019;45:1542-50.

18. Srinivasa DR, Holland M, Sbitany H. Optimizing perioperative strategies to maximize success with prepectoral breast reconstruction. Gland Surg 2019;8:19-26.

19. Jones G, Antony AK. Single stage, direct to implant prepectoral breast reconstruction. Gland Surg 2019;8:53-60.

20. Casella D, Di Taranto G, Marcasciano M, et al. Evaluation of prepectoral implant placement and complete coverage with TiLoop bra mesh for breast reconstruction: a prospective study on long-term and patient-reported BREAST-Q outcomes. Plast Reconstr Surg 2019;143:1e-9e.

21. Gabriel A, Sigalove S, Sigalove NM, et al. Effect of Body Mass Index on Outcomes after Prepectoral Breast Reconstruction. Plast Reconstr Surg 2019;144:550-8.

22. Sinnott CJ, Persing SM, Pronovost M, et al. Impact of postmastectomy radiation therapy in prepectoral versus subpectoral implant-based breast reconstruction. Ann Surg Oncol 2018;25:2899-908. 